\title{
An efficient technique for solving the arbitrarily multilayered electrostatic problems with singularity arising from a degenerate boundary
}

\author{
Shiang-Woei Chyuan ${ }^{1}$, Yunn-Shiuan Liao ${ }^{1}$ and Jeng-Tzong Chen ${ }^{2}$ \\ ${ }^{1}$ Department of Mechanical Engineering, National Taiwan University, Taipei, Taiwan, \\ Republic of China \\ ${ }^{2}$ Department of Harbor and River Engineering, National Taiwan Ocean University, Keelung, \\ Taiwan, Republic of China \\ E-mail: yeaing@iris.seed.net.tw
}

Received 6 February 2004

Published 30 July 2004

Online at stacks.iop.org/SST/19/R47

doi:10.1088/0268-1242/19/9/R02

\begin{abstract}
Engineers usually adopt multilayered design for semiconductor and electron devices, and an accurate electrostatic analysis is indispensable in the design stage. For variable design of electron devices, the BEM has become a better method than the domain-type FEM because BEM can provide a complete solution in terms of boundary values only, with substantial saving in modelling effort. Since dual BEM still has some advantages over conventional BEM for singularity arising from a degenerate boundary, the dual BEM accompanied by subregion technology, instead of tedious calculation of Fourier-Bessel transforms for the spatial Green's functions, was used to efficiently simulate the electric effect of diverse ratios of permittivity between arbitrarily multilayered domain and the fringing field around the edge of conductors. Results show that different ratios of permittivity will affect the electric field seriously, and the values of surface charge density on the edge of conductors are much higher than those on the middle part because of fringing effect. In addition, if using the DBEM to model the fringing field around the edge of conductors, the minimum allowable data of dielectric strength for keeping off dielectric breakdown can be obtained very efficiently.
\end{abstract}

(Some figures in this article are in colour only in the electronic version)

\section{Introduction}

Electrostatics, as used here, is the term given to the study of the interactions between electrically charged bodies. Severely speaking electrostatics includes the study of the forces which hold individual electrons and ions together to form atoms, the chemical forces which bind the atoms together and much of the science of plasma physics and electrochemistry [1]. When device dimensions are much less than the wavelength of electromagnetic radiation at a particular frequency, then the response of the system at that frequency can be considered quasistatic in that the emission, transmission, or absorption of electromagnetic radiation can be ignored [2]. For many microelectromechanical systems (MEMS) and electron devices, knowledge of the electric potential $(V)$ and 
electric field intensity $(\boldsymbol{E})$ are very important [3]. Therefore, electrical engineers are familiar with electrostatic problems, and diverse numerical methods have been regularly used in MEMS and EM (electromagnetics) [4]. Among different numerical approaches, finite element method (FEM), which is based on the representation and approximate solution of boundary value problems of engineering mathematics in terms of partial differential equations [5], and boundary element method (BEM) based on integral equations [6] have moved from being research tools for scientists to become powerful design tools for engineers. One of the main advantages of BEM, when compared to FEM, is that discretizations are restricted only to the boundaries, making data generation much easier. The BEM is also ideally suited to the analysis of external problems where domains extend to infinity, since discretizations are confined to the internal boundaries with no need to truncate the domain at a finite distance and impose artificial boundary conditions, and to problems involving some form of discontinuity or singularity, due to the use of singular fundamental solutions as test functions. Especially for variable design of electron devices, many laborious works of finite element modelling compared to those of boundary element model are needed because BEM can provide a complete solution in terms of boundary values only, with substantial saving in modelling effort. Therefore, there is no doubt that BEM has become a very appealing approach in numerical simulation of EM and MEMS [7-12] even if many engineers still use commercial package and waste much time to set up adverse FEM models in the design stage nowadays.

Because modern MEMS and electron devices design usually contains very thin conducting plates (e.g. a parallelplate capacitor), the singularity problems arising from a degenerate boundary (The degenerate boundary refers to a boundary, two portions of which approach each other such that the exterior region between the two portions becomes infinitely thin.) are frequently formed, and it is well known that the coincidence of the boundaries gives rise to an ill-conditioned problem. The sub-domain technique in conventional BEM with artificial boundaries for degenerate boundary has been introduced to ensure a unique solution. The main drawback of the technique is that the deployment of artificial boundaries is arbitrary and, thus, cannot be implemented easily into an automatic procedure. In addition, model creation is more troublesome than in the single domain approach. To tackle such degenerate boundary electrostatic problems, dual BEM (DBEM) has been proposed in [13], and the above-mentioned boundary value problems can be solved efficiently in the original single domain if using DBEM.

The paper is organized as follows. Section 2 involves the reviews of electrostatic Green's functions in layered dielectrics. In section 3, we briefly introduce the procedure of dual boundary integral equation for electrostatic problems. Numerical results are provided and compared in section 4 to establish the suitability and accuracy of the DBEM. Some remarks based on the reported results were discussed in section 5. Finally, there is a concise conclusion in section 6 .

\section{Reviews of electrostatic Green's functions in layered dielectrics}

Generally the multilayered medium structure has been recently developed and designed in many microwave circuits to provide high-cost performance as commercial products, accurate and expedient prediction of electric performance of high-speed interconnects is critically dependent on efficient simulation of capacitance and inductance of layered dielectric substrates. While using the integral equation formulations formulated by the boundary integral equation techniques for solving electrostatic problems, how to search for a Green's function is seemed to be the first and most important thing.

For the case of layered substrates, commonly utilized techniques use one of two types of Green's functions. The first one is used as kernel of the integral equation (the freespace Green function), in which case, polarization charges on the dielectric interfaces need to be introduced as unknowns $[14,15]$. In $[14,15]$, the spatial domain approach using the free-space Green function has been developed to calculate the capacitance and inductance matrices of multiconductor transmission lines located arbitrarily in a multilayered medium of finite extent. The potential in the medium is expressed in terms of the free charge at the conductor-dielectric interfaces and the total charge at the dielectric-dielectric interfaces. The second one is called the spatial Green function which is much more complicated than the free-space Green function because it is consistent with the boundary conditions at the dielectric interfaces [16-19]. Comparing the free-space Green function with spatial Green's function, one can see that: (1) although the free-space Green's function is simple, the number of unknowns involved in the discrete problem is large. (2) For spatial Green's function, the only polarization charges required as additional unknowns are those at any finite dielectric boundaries and the number of unknowns in this case is reduced significantly. (3) Even though closed-form expressions in terms of an infinite series are available for the cases of single-layer microstrip and stripline configurations, the calculation of spatial Green's functions for multilayered substrates requires the computationally tedious calculation of Fourier-Bessel transforms because it is necessary to relate the Fourier coefficients in each layer to the boundary conditions on the interfaces of the dielectric layers.

Because there are also many difficulties for irregular geometrical design while using the afore-mentioned conventional free-space and spatial Green's functions, a systematic and robust methodology is needed for solving the arbitrarily multilayered electrostatic problems. However, many difficulties will still be faced if using DBEM for the multilayered domain because we also need more external boundary conditions at the interface between two dielectric media, which should be calculated. Since electromagnetic problems often involve media with different physical properties and require knowledge of the relation of the field quantities at the interface between two media, the DBEM accompanied by subregion technique was successfully used to determinate how the $\boldsymbol{E}$ and $\boldsymbol{D}(\boldsymbol{D}=\varepsilon \boldsymbol{E}$, electric flux density) vectors change in crossing the interface in this paper. For electrical engineering practices, since the numbers of elements and nodes for FEM are much higher than those of DBEM to get a reasonable result, the present DBEM accompanied by the subregion technique has great potential in multilayered electrostatic problems for future industrial applications. 


\section{Dual boundary integral equations}

For a homogeneous medium, the governing equation of electrostatics can be written in the following form,

$$
\nabla^{2} \boldsymbol{V}=-\rho / \varepsilon
$$

where $\nabla^{2}$ is the Laplacian operator. Equation (1) is known as Poisson's equation; it states that the divergence of the gradient of electric potential $(V)$ equals $-\rho / \varepsilon$ for a simple medium, where $\varepsilon$ is the permittivity of the medium and $\rho$ is the volume density of free charges. At points in a simple medium where there is no free charge, equation (1) is reduced to

$$
\nabla^{2} \boldsymbol{V}=0
$$

which is known as Laplace's equation. Equation (2) plays a very important role in MEMS and EM. It is the governing equation for electrostatic problems involving a set of conductors, such as capacitors, maintained at different potentials. Once $V$ is found from equation (2), $\boldsymbol{E}$ can be determined from $-\nabla V$, and the charge distribution on the conductor surfaces can be determined from surface charge density $\rho_{s}=\varepsilon E_{n}$.

The electrostatic problem consists of finding the unknown potential function $\Phi$ (or $V$ ) in the partial differential equation. In addition to the fact that $\Phi$ satisfies equation (2) within a prescribed solution region $\Omega$, the potential function $\Phi$ must satisfy certain conditions on $B$ which is the boundary of $\Omega$. Usually these boundary conditions are the Dirichlet and Neumann types. Therefore, the governing equation and boundary conditions of electrostatic problems could be written in the following form.

Governing equation:

$$
\nabla^{2} \Phi(x)=0, \quad x \text { in } \Omega
$$

Dirichlet boundary condition:

$$
\Phi(x)=f(x), \quad x \text { on } B
$$

Neumann boundary condition:

$$
\partial \Phi(x) / \partial n_{x}=g(x), \quad x \text { on } B,
$$

where $f(x)$ and $g(x)$ denote the known boundary data, and $n_{x}$ is the unit outer normal vector at the point $x$ on the boundary.

Based on the dual boundary integral equation formulation for electrostatic problem, we have

$$
\begin{aligned}
& \alpha \Phi(x)=\operatorname{CPV} \int_{B} T(s, x) \Phi(s) \mathrm{d} B(s) \\
& -\operatorname{RPV} \int_{B} U(s, x)\left[\partial \Phi(s) / \partial n_{s}\right] \mathrm{d} B(s)
\end{aligned}
$$

$$
\begin{gathered}
\alpha\left[\partial \Phi(x) / \partial n_{x}\right]=\mathrm{HPV} \int_{B} M(s, x) \Phi(s) \mathrm{d} B(s) \\
-\mathrm{CPV} \int_{B} L(s, x)\left[\partial \Phi(s) / \partial n_{s}\right] \mathrm{d} B(s),
\end{gathered}
$$

where the kernel functions, $U(s, x)=\ln (r), T(s, x)=$ $\partial U(s, x) / \partial n_{s}, \quad L(s, x)=\partial U(s, x) / \partial n_{x}, \quad M(s, x)=$ $\partial^{2} U(s, x) / \partial n_{x} \partial n_{s}, \boldsymbol{r}=|s-\boldsymbol{x}|, \boldsymbol{s}$ and $\boldsymbol{x}$ being the position vectors of the points $s$ and $x$, respectively, and $n_{s}$ is the unit outer normal vector at point $s$ on the boundary (see figure 1). In addition, RPV is the Riemann principal value, CPV is the Cauchy principal value, HPV is the Hadamard principal

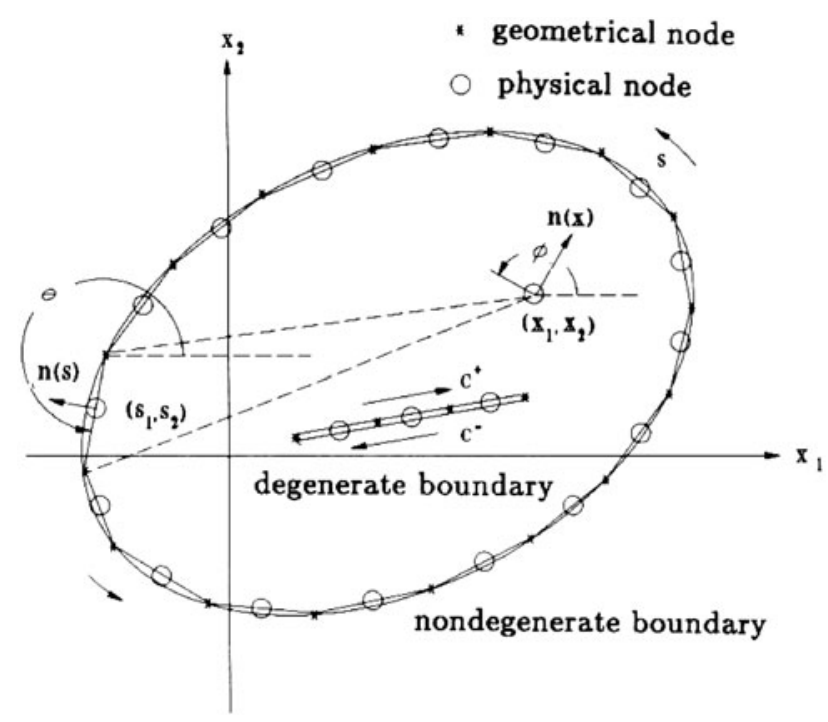

Figure 1. Boundary element discretization for degenerate boundary and non-degenerate boundary.
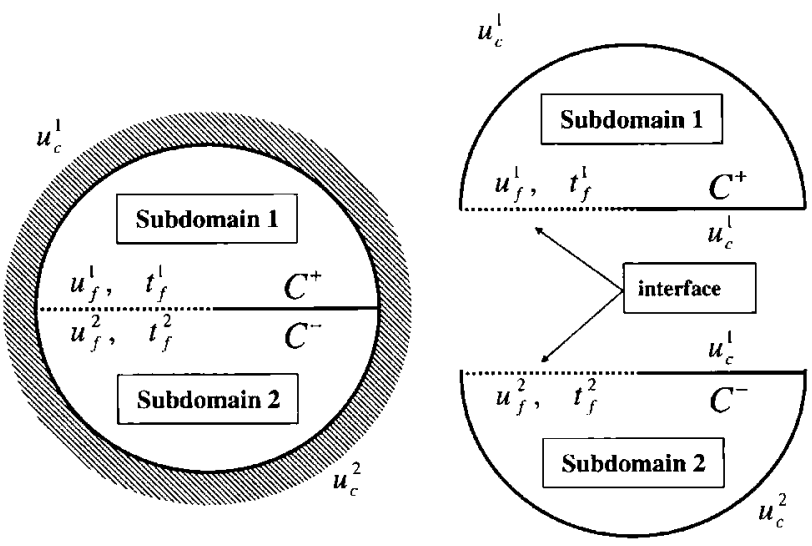

Figure 2. A figure sketch of the multi-domain.

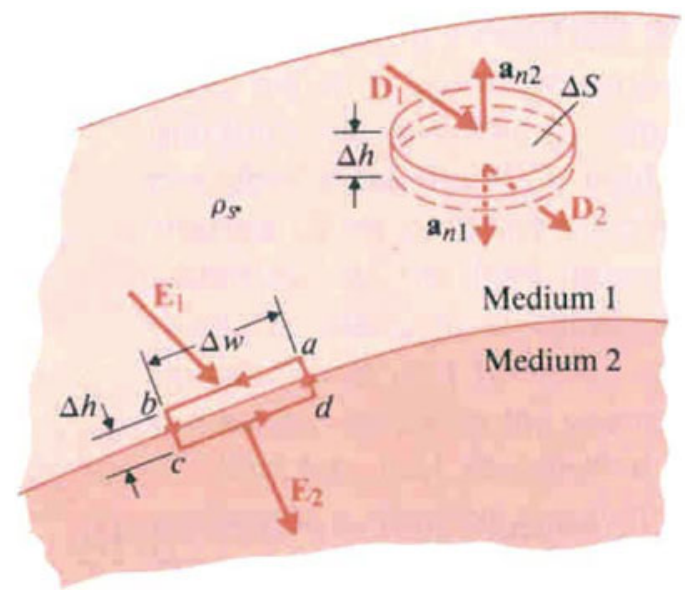

Figure 3. An interface between two media.

value, and $\alpha$ depends on the collocation point ( $\alpha=2 \pi$ for an interior point, $\alpha=\pi$ for a smooth boundary, $\alpha=0$ for an exterior point). The commutativity property of the trace operator and the normal derivative operator provides us with alternative ways to calculate the Hadamard principal value 
analytically [12]. First, L'Hospital's rule is employed in the limiting process. Second, the normal derivative of the Cauchy principal value should be taken carefully by using Leibnitz' rule, and then the finite part can be obtained. The finite part has been termed the Hadamard principal value or Mangler's principal value. In the derivation of dual equations, two alternatives can be applied to determine the Hadamard principal value as presented in appendix A. Generally, equation (6) is called singular boundary integral equation, and equation (7) is called hypersingular boundary integral equation. Since the hypersingular equation plays an important role in the degenerate problems, many researchers have paid much attention to this. After discretizing the boundary into $2 N$ boundary elements, equations (6) and (7) are reduced to

$$
\begin{aligned}
& {[U]_{2 N \times 2 N}\{t\}_{2 N \times 1}=[T]_{2 N \times 2 N}\{u\}_{2 N \times 1}} \\
& {[L]_{2 N \times 2 N}\{t\}_{2 N \times 1}=[M]_{2 N \times 2 N}\{u\}_{2 N \times 1}}
\end{aligned}
$$

where $[U],[T],[L]$ and $[M]$ are the four influence matrices, $\{u\}$ and $\{t\}$ are the boundary data for the primary and the secondary boundary variables: $\{\Phi\}$ and $\{\partial \Phi / \partial n\}$, respectively. These above-mentioned four influence matrices for interior and exterior fields are presented in appendix B. Generally for electrostatic problems without degenerate scale, the aforementioned influence matrix $[U]$ is nonsingular, either equation (8) or equation (9) can be solved by Gaussian elimination and $L U$ decomposition very well. But for degenerate scale problem, $[U]$ matrix is singular and the rank is deficient, then the SVD (singular value decomposition) technique need to be used [20]. For multilayered electrostatic problems, the DBEM accompanied by subregion technique for multi-domain is needed, and the methods are presented in appendix $\mathrm{C}$.

\section{DBEM simulation of multilayered electrostatic problems}

In order to demonstrate the suitability and efficiency of DBEM presented in this paper, two electrostatic problems were used. Basically for the nondegenerate boundary problems like the following case 1 , either singular BEM (just using the first kind kernels of $U(s, x)$ and $T(s, x)$ ), hypersingular BEM (the second kind kernels of $L(s, x), M(s, x))$ or DBEM (using the first kind kernels of $U(s, x), T(s, x)$ and the second kind kernels of $L(s, x), M(s, x)$ in the meantime) accompanied by subregion technology can be used. But for the multilayered electrostatic problems with degenerate boundary like the following case 2, the DBEM accompanied by subregion technology plays an important role, and conventional BEM (singular BEM or hypersingular BEM) without external artificial boundaries cannot be used.

Case 1. Consider the potential problem shown in figure 4 . The potentials at $x=0, x=a$ and $y=0$ sides are zero while the potential at $y=b$ side is $V_{0}$. Try to find the potential distribution in the multilayered domain.

Because it is not easy to obtain analytical data, FEM was used to calculate the reference data [21]. For convenience, the values of $a, b$ and $c$ are assumed to be 30,20 and $10 \mu \mathrm{m}$. respectively. Four points will be analysed using rough mesh discretization (72 elements and 72 nodes, see

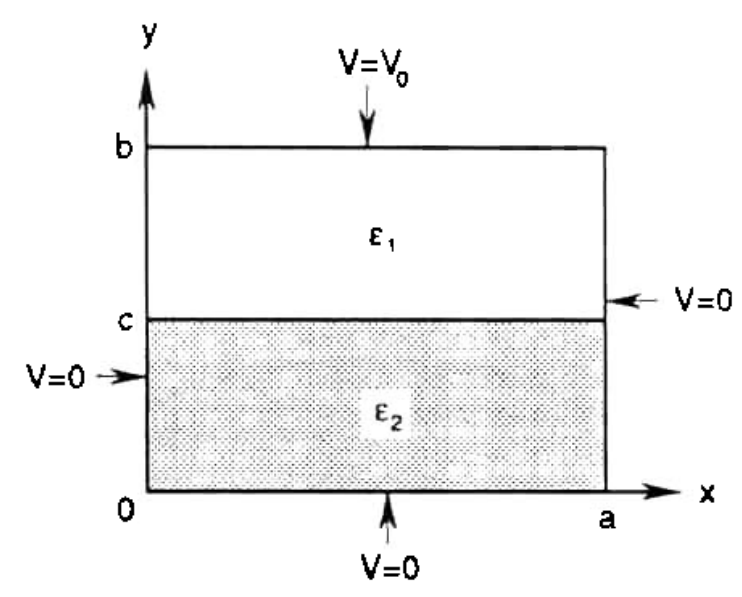

Figure 4. Figure of case 1 (without degenerate boundary).

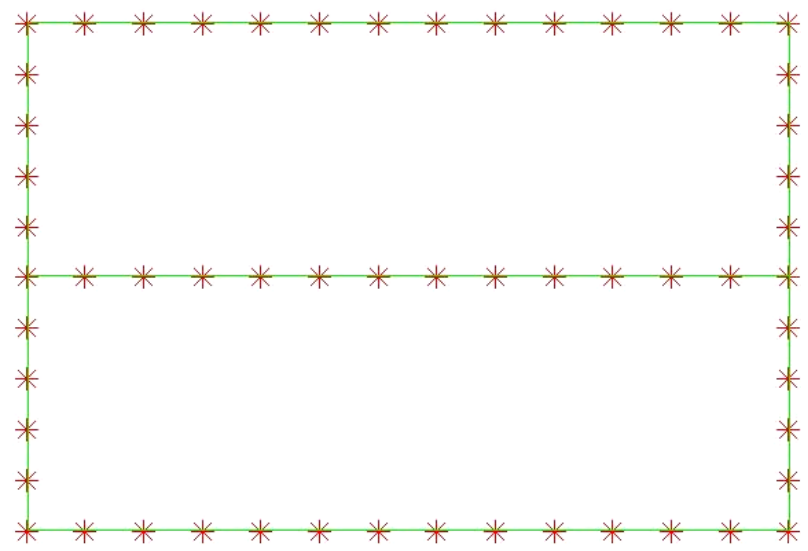

Figure 5. The related DBEM mesh discretization of case 1 (without degenerate boundary).

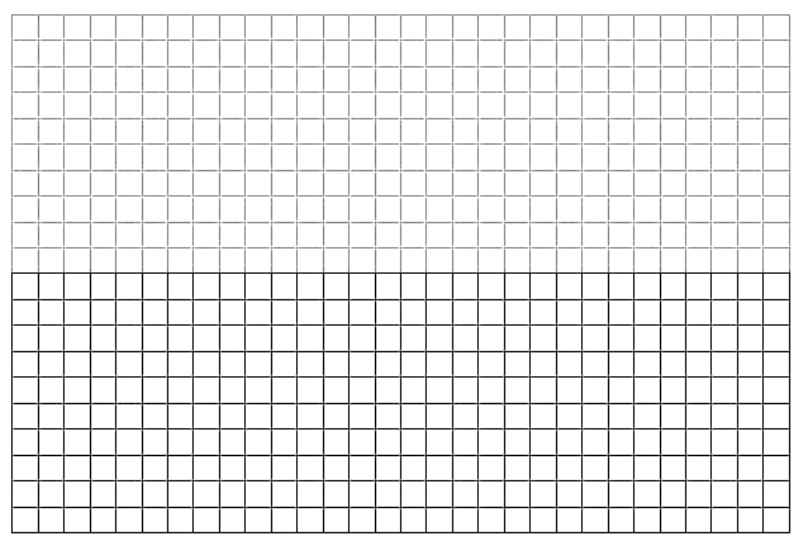

Figure 6. The related FEM mesh discretization of case 1.

figure 5) of conventional DBEM (or BEM) accompanied by subregion technology, and compared with reference data computed from a larger FEM model (600 elements and 651 nodes, see figure 6). The results of electric potential under diverse numerical methods were listed in tables 1 and 2 and shown in figures 7 and 8. Comparing the results of electric potential field (equipotential lines) using DBEM and FEM (see figures 7 and 8), one can see that the difference of electric potential distribution is very little. Therefore, DBEM (or BEM) accompanied by subregion technology used in this 

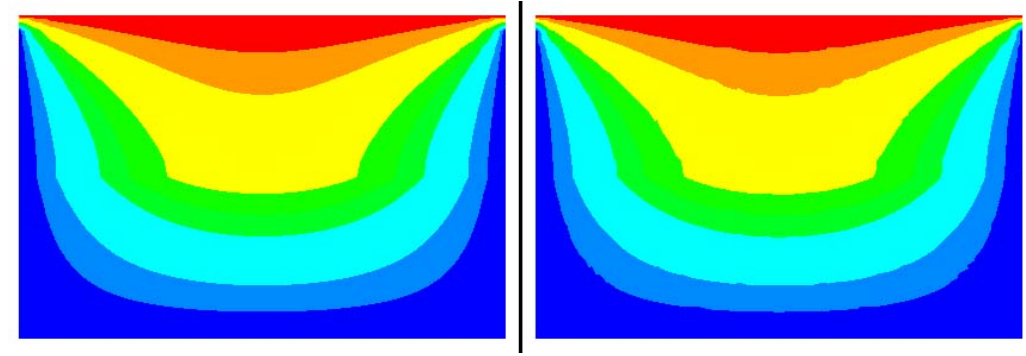

Figure 7. The results of electric potential $(V)$ (equipotential lines; ranging from $+V_{0}$ at top (red in online edition) to 0 at bottom (dark blue online)) of case $1(R=10)$ if using DBEM (left part) and FEM (right part).
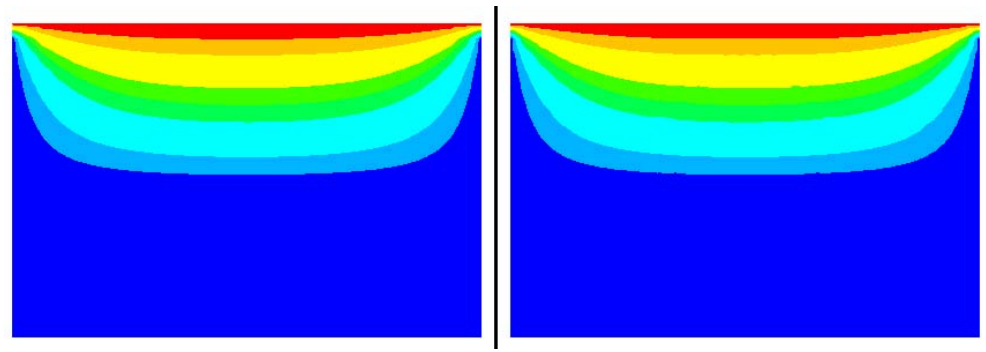

Figure 8. The results of electric potential $(V)$ (equipotential lines; ranging from $+V_{0}$ at top (red in online edition) to 0 at bottom (dark blue online)) of case $1(R=0.1)$ if using DBEM (left part) and FEM (right part).

Table 1. The results of electric potential $(V)$ of case $1(R=10)$ if using the conventional BEM, DBEM and FEM.

\begin{tabular}{llllll}
\hline $\begin{array}{l}\text { Locations } \\
(x, y)\end{array}$ & $\begin{array}{l}\text { Results from } \\
\text { singular BEM } \\
U, T \text { kernels }\end{array}$ & $\begin{array}{l}\text { Results from } \\
\text { hypersingular BEM } \\
L, M \text { kernels }\end{array}$ & $\begin{array}{l}\text { Results from } \\
\text { DBEM } \\
U, T, L, M \text { kernels }\end{array}$ & $\begin{array}{l}\text { Results from } \\
\text { FEM }\end{array}$ & $\begin{array}{l}\text { Difference } \\
\text { between } \\
\text { DBEM and FEM }\end{array}$ \\
\hline$(18.0,3.00)$ & $0.17302 V_{0}$ & $0.18490 V_{0}$ & $0.17302 V_{0}$ & $0.1743103 V_{0}$ & $-0.74 \%$ \\
$(4.00,9.00)$ & $0.27448 V_{0}$ & $0.28312 V_{0}$ & $0.27448 V_{0}$ & $0.2809692 V_{0}$ & $-2.31 \%$ \\
$(25.0,16.0)$ & $0.59607 V_{0}$ & $0.63756 V_{0}$ & $0.59607 V_{0}$ & $0.6000305 V_{0}$ & $-0.67 \%$ \\
$(5.00,17.0)$ & $0.67492 V_{0}$ & $0.71273 V_{0}$ & $0.67492 V_{0}$ & $0.679071 V_{0}$ & $-0.61 \%$ \\
\hline
\end{tabular}

Table 2. The results of electric potential $(V)$ of case $1(R=0.1)$ if using the conventional BEM, DBEM and FEM.

\begin{tabular}{llllll}
\hline $\begin{array}{l}\text { Locations } \\
(x, y)\end{array}$ & $\begin{array}{l}\text { Results from } \\
\text { singular BEM } \\
\mathrm{U}, \mathrm{T} \text { kernels }\end{array}$ & $\begin{array}{l}\text { Results from } \\
\text { hypersingular BEM } \\
\text { L, M kernels }\end{array}$ & $\begin{array}{l}\text { Results from } \\
\text { DBEM } \\
\mathrm{U}, \mathrm{T}, \mathrm{L}, \mathrm{M} \text { kernels }\end{array}$ & $\begin{array}{l}\text { Results from } \\
\text { FEM }\end{array}$ & $\begin{array}{l}\text { Difference } \\
\text { between } \\
\text { DBEM and FEM }\end{array}$ \\
\hline$(18.0,3.00)$ & $0.017302 V_{0}$ & $0.018490 V_{0}$ & $0.017302 V_{0}$ & $0.01741943 V_{0}$ & $0.67 \%$ \\
$(4.00,9.00)$ & $0.027448 V_{0}$ & $0.028312 V_{0}$ & $0.027448 V_{0}$ & $0.02810059 V_{0}$ & $-2.32 \%$ \\
$(25.0,16.0)$ & $0.480640 V_{0}$ & $0.511030 V_{0}$ & $0.480640 V_{0}$ & $0.48833313 V_{0}$ & $-1.58 \%$ \\
$(5.00,17.0)$ & $0.589690 V_{0}$ & $0.615380 V_{0}$ & $0.589690 V_{0}$ & $0.5929200 V_{0}$ & $-0.54 \%$ \\
\hline
\end{tabular}

paper seems to be a very efficiently numerical method for the multilayered electrostatic problems without degenerate boundary (e.g. thin strip conductors). From the results of electric potential $V(x, y)$ listed in tables 1 and 2 , one can see that the differences between FEM and DBEM are lower than $2.4 \%$. In addition, both singular BEM and hypersingular BEM accompanied by subregion technology can deal with this nondegenerate boundary problem well.

In order to investigate the effect of diverse ratios of permittivity ( $R=\varepsilon_{1} / \varepsilon_{2}$ ) between subdomains 1 and 2 , the results of electric potentials of $Y=10 \mu \mathrm{m}$ interface line and $X=15 \mu \mathrm{m}$ lines from DBEM are shown in figures 9 and 10. From figures 9 and 10, one can see that the values of $R$ seriously affect the distribution of voltages and engineers can adjust $R$ according to their needs.
Case 2. Similar to case 1, but insert two thin strip conductors (length $=10 \mu \mathrm{m}$ ) into the centre part of these two media shown in figure 4 . If the voltage of the upper strip conductor is 0 and that of the lower one is $V_{0}$, determine the electric field and charge distributions.

Because these two strip conductors form a new degenerate boundary and a closed contour formed by two lines $C^{+}$and $C^{-}$ (see figure 1) for the degenerate boundary, the singular BEM or hypersingular BEM without external artificial interface cannot cope with this degenerate case, but the electric potential can be calculated just in one run if using DBEM. Similar to case 1, FEM was also used to calculate the reference data, but the linear shape function of finite element model shown in figure 6 was replaced by parabolic shape function in order to model the fringing effect near the edge of these two strip 


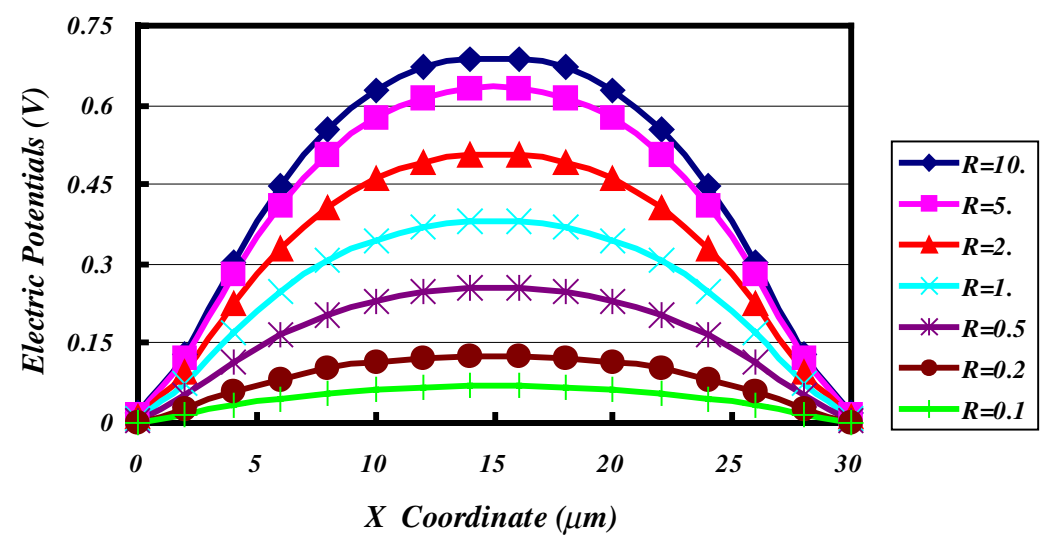

Figure 9. Results of electric potentials $(V)$ of $Y=10 \mu \mathrm{m}$ interface line under diverse ratios of permittivity $(R)$ between subdomains 1 and 2-case 1 (unit: $V_{0}$ ).

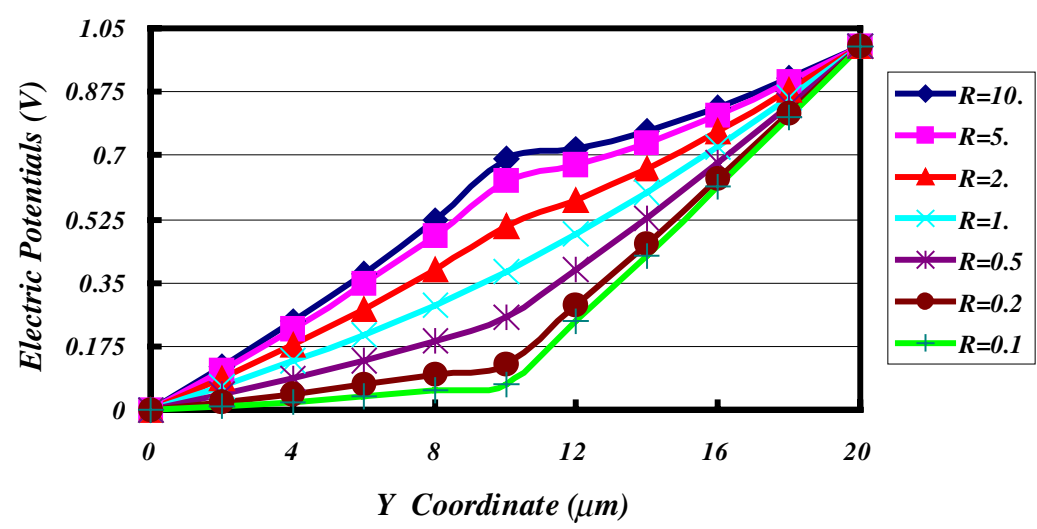

Figure 10. Results of electric potentials $(V)$ of $X=15 \mu \mathrm{m}$ line under diverse ratios of permittivity $(R)$ between subdomains 1 and 2 - case 1 (unit: $V_{0}$ ).

conductors. Four points will also be analysed using more dense mesh discretization (180 elements and 156 nodes, see figure 11) of DBEM accompanied by subregion technology, and compared with reference data computed from a larger FEM model (600 elements and 1901 nodes). The results of electric potential under diverse numerical methods were listed in table 3 and shown in figure 12. Comparing the results of electric potential field of DBEM with those of FEM (see figure 12), one can see that the difference of electric potential distribution is also very little and the fringing effect near the edge of these two strip conductors can be modelled very well.

From the results of electric potential $V(x, y)$ listed in table 3 and shown in figure 12, one can find that (1) the conventional BEM without external artificial boundaries cannot solve the electrostatic problem with degenerate boundaries such as this case. (2) DBEM accompanied by subregion technology can analyse the multilayered electrostatic problem with degenerate boundary very efficiently. (3) The fringing effect near the edge of these two strip conductors can be simulated very well using DBEM. (4) The differences between FEM and DBEM are lower than $5.0 \%$. In addition, the results of electric potentials of $Y=10 \mu \mathrm{m}$ interface line and $X=15 \mu \mathrm{m}$ lines from DBEM are also shown in figures 13 and 14 in order to investigate the effect of diverse ratios of permittivity $(R)$ between subdomains 1 and 2 . From figures 13 and 14 , one can see that the values of $R$ also critically influence on the distribution of electric

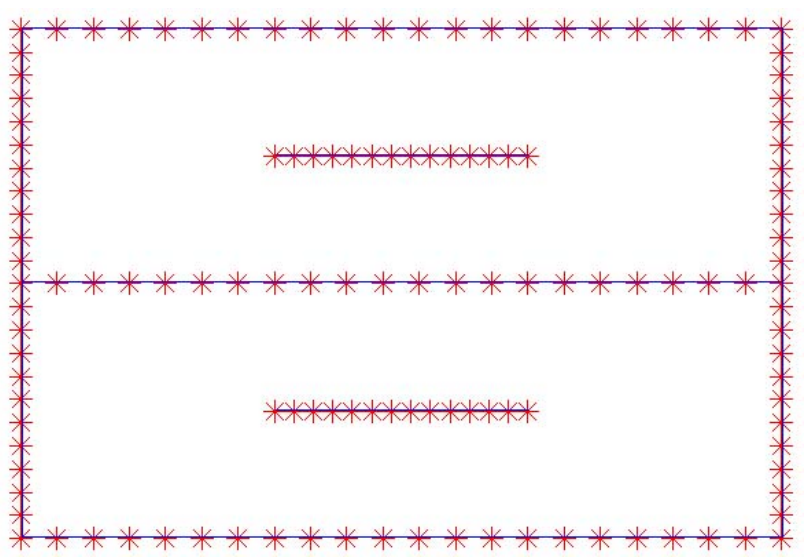

Figure 11. The related DBEM mesh discretization of case 2 (with degenerate boundary).

potentials like case 1 (see figures 9 and 10). Comparing with the results of nondegenerate case, the electric potential field of degenerate case is more complicated because of degenerate boundary.

Besides electric potentials, the distributions of normal electric field $\left(E_{n}\right)$ on the top and bottom sides of upper conductor under diverse $R$ can be shown in figures 15 and 16. From figure 16 , one can see that the values of $E_{n, 2}$ on the 

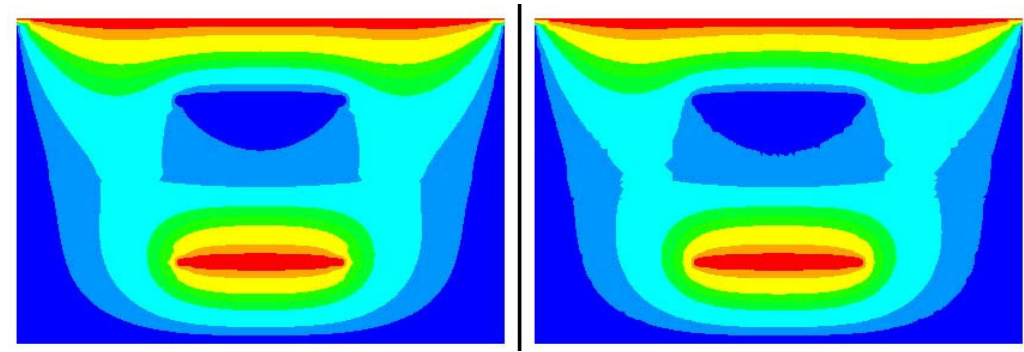

Figure 12. The results of electric potential $(V)$ (equipotential lines; ranging from $+V_{0}$ at top and lower middle area (red in online edition) to 0 at bottom and upper middle area (dark blue online)) of case $2(R=10)$ if using DBEM (left part) and FEM (right part).

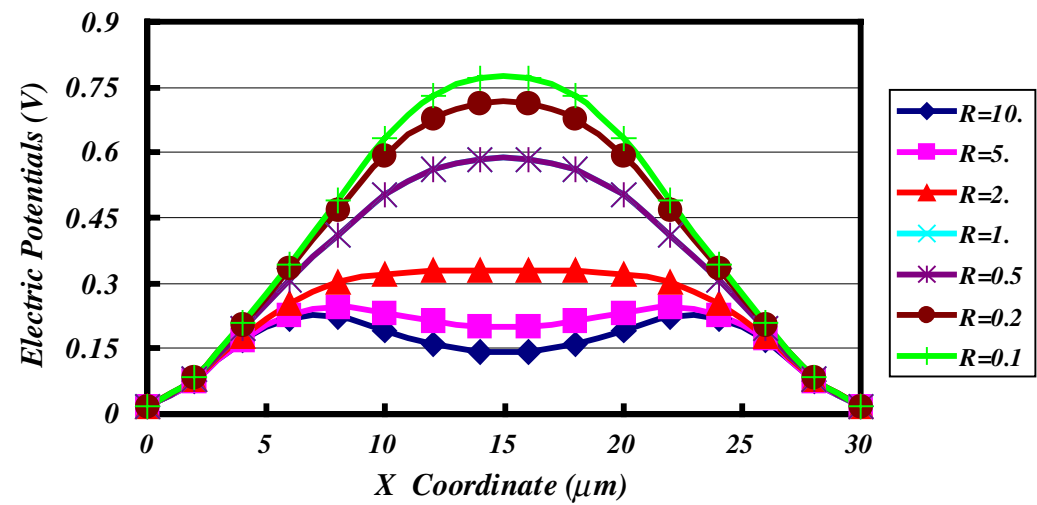

Figure 13. Results of electric potentials $(V)$ of $Y=10 \mu \mathrm{m}$ interface line under diverse ratios of permittivity $(R)$ between subdomains 1 and 2-case 2 (unit: $V_{0}$ ).

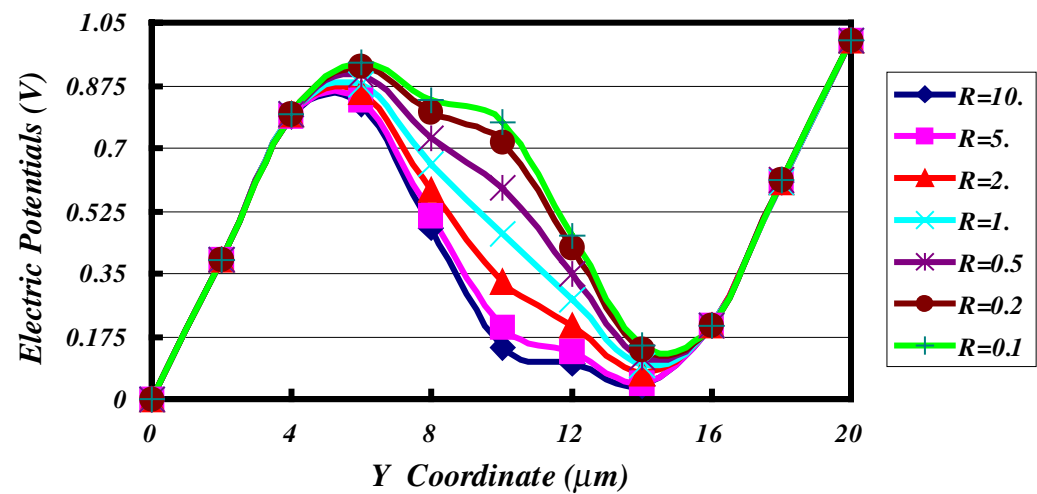

Figure 14. Results of electric potentials $(V)$ of $X=15 \mu \mathrm{m}$ line under diverse ratios of permittivity $(R)$ between subdomains 1 and 2 - case 2 (unit: $V_{0}$ ).

Table 3. The results of electric potential $(V)$ of case $2(R=10)$ if using the conventional BEM, DBEM and FEM.

\begin{tabular}{llllll}
\hline $\begin{array}{l}\text { Locations } \\
(x, y)\end{array}$ & $\begin{array}{l}\text { Results from } \\
\text { singular BEM } \\
U, T \text { kernels }\end{array}$ & $\begin{array}{l}\text { Results from } \\
\text { hypersingular BEM } \\
L, M \text { kernels }\end{array}$ & $\begin{array}{l}\text { Results from } \\
\text { DBEM } \\
U, T, L, M \text { kernels }\end{array}$ & $\begin{array}{l}\text { Results from } \\
\text { FEM }\end{array}$ & $\begin{array}{l}\text { Difference } \\
\text { between } \\
\text { DBEM and FEM }\end{array}$ \\
\hline$(24.0,16.5)$ & NA & NA & $0.52181 V_{0}$ & $0.5144898 V_{0}$ & $1.42 \%$ \\
$(6.50,12.0)$ & NA & NA & $0.23801 V_{0}$ & $0.2301575 V_{0}$ & $3.41 \%$ \\
$(22.5,6.00)$ & NA & NA & $0.34638 V_{0}$ & $0.3638855 V_{0}$ & $-4.81 \%$ \\
$(4.00,3.50)$ & NA & NA & $0.10623 V_{0}$ & $0.1108643 V_{0}$ & $-4.18 \%$ \\
\hline
\end{tabular}

bottom side of upper conductor are obviously dependent on the values of $R$ and the location to the left corner of conductor $\left(\operatorname{loc}_{x}\right)$, and the smaller the $R$ is, the larger the $E_{n, 2}$ is. Unlike $E_{n, 2}$, the values of $E_{n, 1}$ on the top side are only apparently counting on the value of $\operatorname{loc}_{x}$, and the effect of the values of
$R$ can be ignored (see figure 15). Similar to the results of upper conductor, the distributions of $E_{n}$ on the top and bottom sides of lower conductor under diverse $R$ can also be shown in figures 17 and 18. From figure 17, one can see that the values of $E_{n, 3}$ on the top side of lower conductor are obviously 


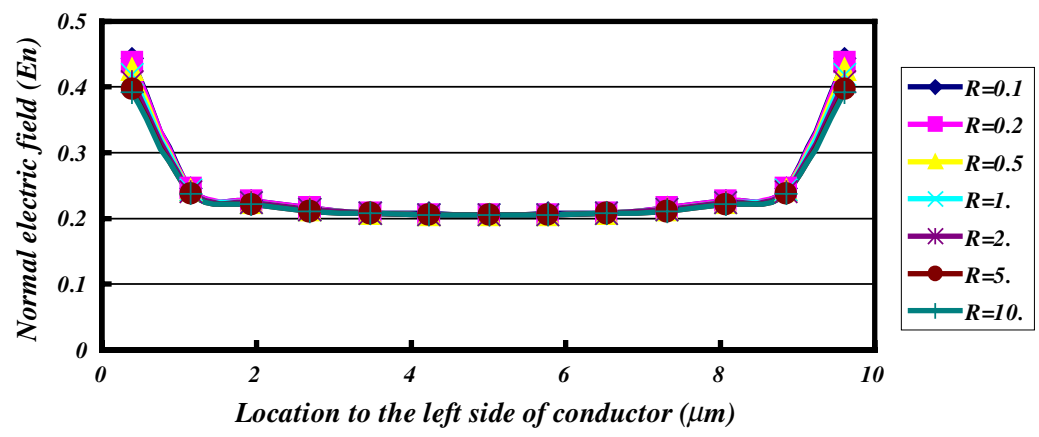

Figure 15. The distribution of normal electric field $\left(E_{n}\right)$ on the top side of upper conductor under diverse ratios of permittivity $(R)$ between subdomains 1 and 2 (unit: $V_{0} / \mu \mathrm{m}$ ).

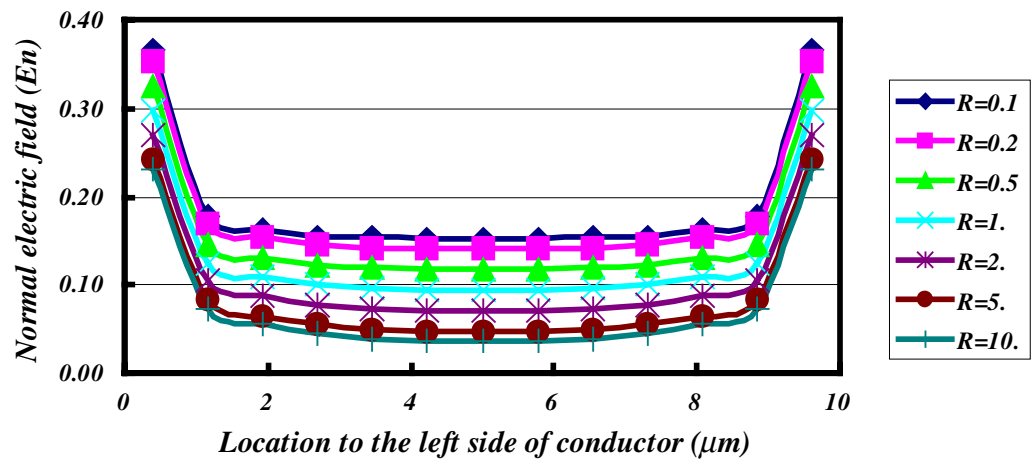

Figure 16. The distribution of normal electric field $\left(E_{n}\right)$ on the bottom side of upper conductor under diverse ratios of permittivity $(R)$ between subdomains 1 and 2 (unit: $V_{0} / \mu \mathrm{m}$ ).

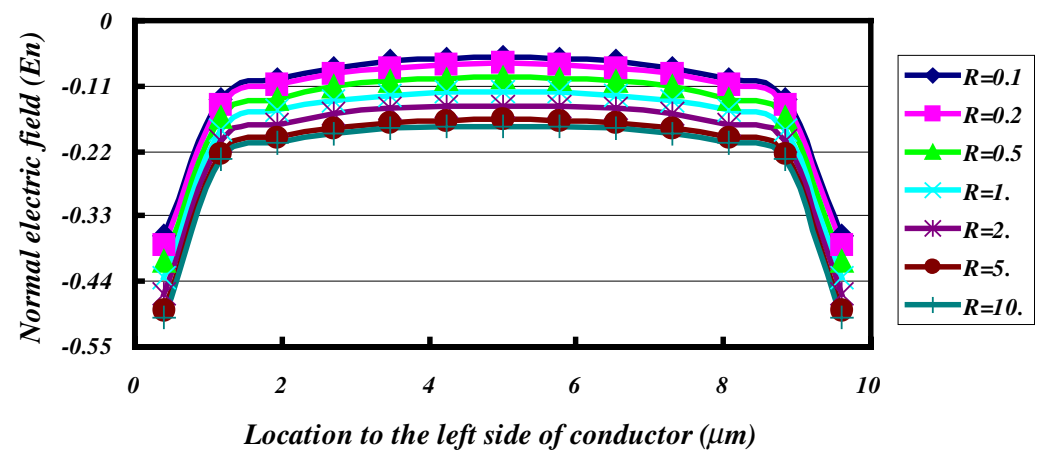

Figure 17. The distribution of normal electric field $\left(E_{n}\right)$ on the top side of lower conductor under diverse ratios of permittivity $(R)$ between subdomains 1 and 2 (unit: $\left.V_{0} / \mu \mathrm{m}\right)$.

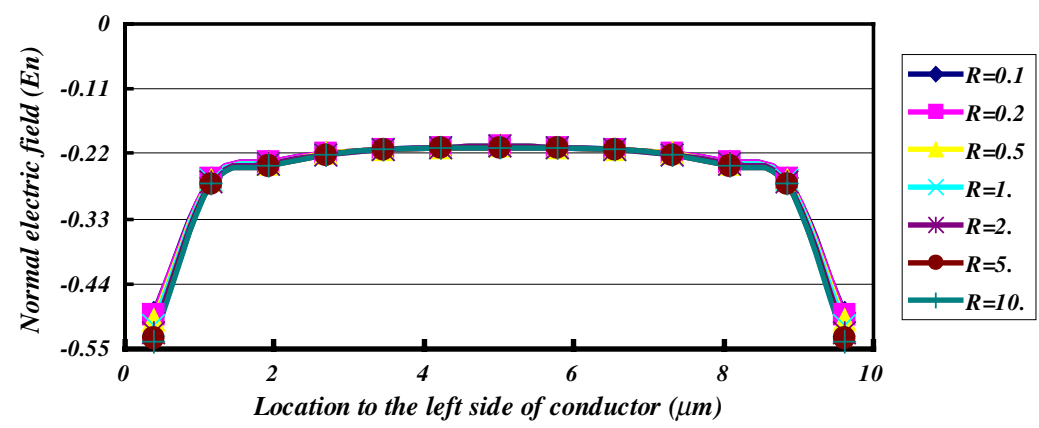

Figure 18. The distribution of normal electric field $\left(E_{n}\right)$ on the bottom side of lower conductor under diverse ratios of permittivity $(R)$ between subdomains 1 and 2 (unit: $V_{0} / \mu \mathrm{m}$ ).

dependent on the values of $R$ and the $\operatorname{loc}_{x}$, but the values of $E_{n, 4}$ on the bottom side are only apparently counting on the value of $\operatorname{loc}_{x}$, and the effect of the values of $R$ can be also neglected (see figure 18). From figures 15 to 18 , one can find that different 


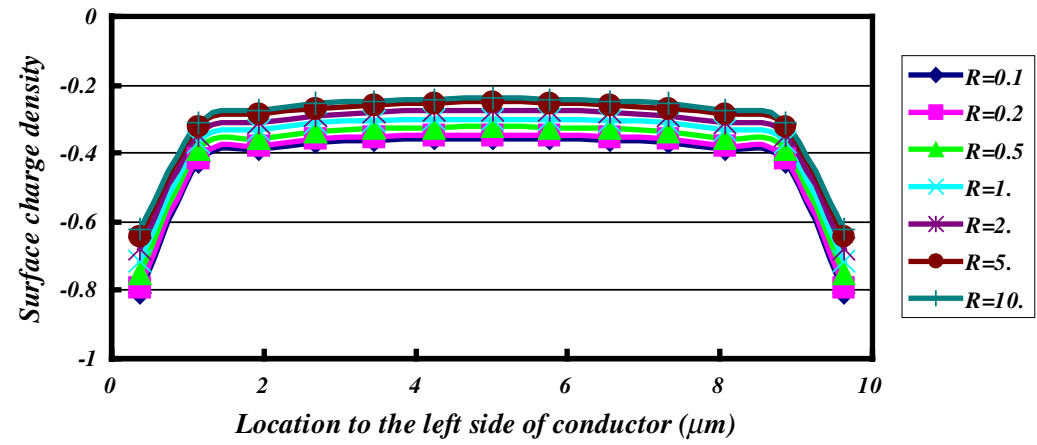

Figure 19. The distribution of surface charge density $\left(\rho_{s}\right)$ of upper conductor under diverse ratios of permittivity $(R)$ between subdomains 1 and 2 (unit: $\varepsilon_{1} V_{0} / \mu \mathrm{m}^{2}$ ).

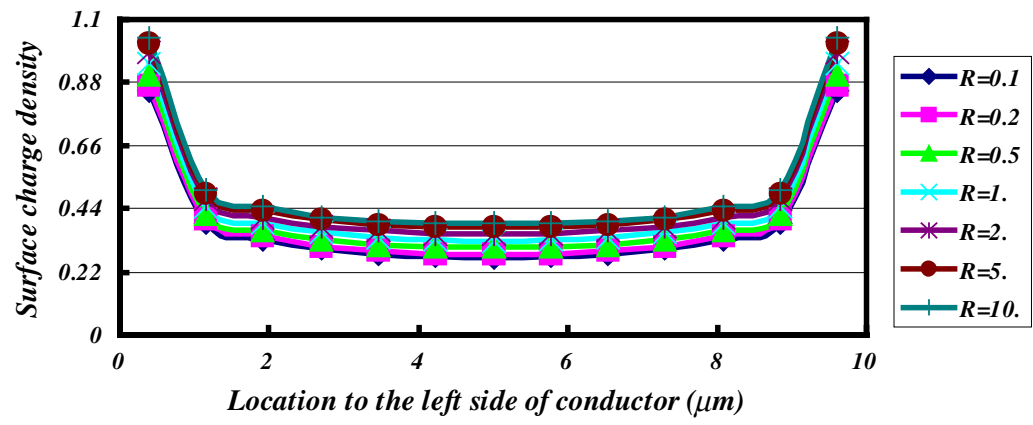

Figure 20. The distribution of surface charge density $\left(\rho_{s}\right)$ of lower conductor under diverse ratios of permottivity $(R)$ between subdomains 1 and 2 (unit: $\varepsilon_{2} V_{0} / \mu \mathrm{m}^{2}$ ).

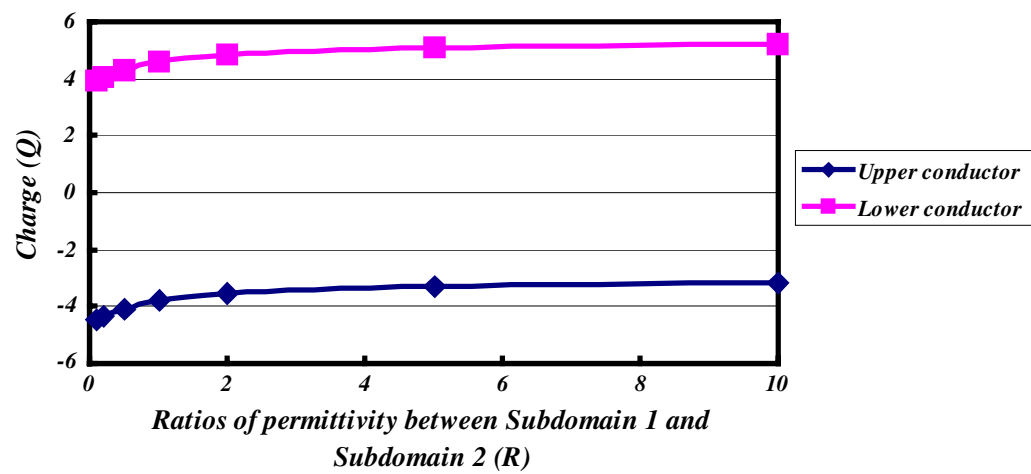

Figure 21. The distribution of charge $(Q)$ on both upper and lower conductors under diverse ratios of permittivity $(R)$ between subdomains 1 and 2 (unit: upper conductor: $\varepsilon_{1} w V_{0}$; lower conductor: $\varepsilon_{2} w V_{0}$ ).

$R$ will affect the electric field seriously, and the values of $E_{n}$ on the edge of conductors are much higher than those on the middle part because of the fringing effect.

As we know the value of $R$ can play a very important role, let us investigate the effect of $R$ for charge distribution. Because the charge distribution on the conductor surfaces can be determined from $\rho_{s}=\varepsilon E_{n}$ (the normal component of the electric field $E_{n}$ at a conductor boundary is equal to the surface charge density $\rho_{s}$ on the conductor divided by the permittivity $\varepsilon[1,2])$ if $\varepsilon$ is a constant. From figures 19 and 20, we can see that the values of surface charge density $\left(\rho_{s}\right)$ on the both upper and lower conductors are obviously dependent on the value of $\operatorname{loc}_{x}$, but not very obvious for the value of $R$. As we all know that the fringing effect around the edge of inserted conductors is as clear as day like $E_{n}$. From these results, we can see the $\rho_{s}$ at the edges becomes much larger than that at the centre for conductors because of fringing effect. If the width of conductor is $w$, the distribution of charge $(Q)$ on both upper and lower conductors under diverse $R$ can be shown in figure 21. From figure 21, we can see that the larger the value of $R$ is, the larger the $Q$ of lower capacitor is, but the larger the value of $R$ is, the less the $Q$ of upper capacitor is.

\section{Discussions}

(1) For the electrostatic problems without degenerate boundary like case 1, singular BEM, hypersingular BEM and DBEM are all very usefully numerical tools. For the electrostatic problems with singularity arising from degenerate boundary such as case 2 , it is well known that the coincidence of the boundaries will give rise to 
an ill-conditioned problem if using conventional BEM. Therefore DBEM presented in this paper became a very efficient solver for modelling the fringing field around the edge of electron devices and the conventional BEM without external artificial boundaries cannot be used for the electrostatic problem with degenerate boundaries.

(2) By way of DBEM, one can see that the values of normal electric field $\left(E_{n}\right)$ on the bottom side of upper and top side of lower conductors are obviously dependent on the values of ratios of permittivity $(R)$ between subdomains 1 and 2 and the location to the left corner of conductor $\left(\operatorname{loc}_{x}\right.$ ), but the values of $E_{n}$ on the top side of upper and bottom sides of lower conductors are only apparently counting on the value of $\operatorname{loc}_{x}$, and the effect of the values of $R$ can be neglected. Results also show that different $R$ will affect the electric potential $(V)$ and field seriously, and the values of electric field and surface charge density $\left(\rho_{s}\right)$ on the edge of conductors are much higher than those on the middle part because of fringing effect.

(3) As we know from the theory of electrostatics [1, 2], the electrons will be pulled out of the molecules completely if the $\boldsymbol{E}$ is very strong, and the electrons will accelerate under the influence of the $E$, collide violently with the molecular lattice structure, then cause permanent dislocations and damage in the material. While avalanche effect of ionization due to collisions occurs, the material will become conducting, and large currents $(I)$ may result. This phenomenon is called a dielectric breakdown, and the maximum electric field intensity that a dielectric material can withstand without breakdown is the dielectric strength of the material (e.g. for air is $3 \times 10^{6} \mathrm{~V} \mathrm{~m}^{-1}$ ). So the accurate and efficient modelling for fringing effect around the edge of electron devices is very important for performance because we need to know the minimum allowable data of dielectric strength for keeping off dielectric breakdown. Therefore, we recommend our DBEM here because it is more simple and efficient than other conventional BEMs.

(4) For the arbitrarily multilayered electrostatic problems with singularity arising from degenerate boundary, DBEM accompanied by subregion technology can efficiently model the physical behaviour of interface between subdomains 1 and 2 . In addition, for variable design of electron devices, the DBEM has become a better method than the domain-type FEM because DBEM can provide a complete solution in terms of boundary values only, with substantial saving in modelling effort. Especially for calculating the $\rho_{s}$ and charge $(Q)$ of conductors according to diverse design, only the part of $E_{n}$ on the surface of conductors needed to be known, then the DBEM can work very well.

\section{Conclusions}

The dual boundary integral formulation accompanied by subregion technology for arbitrarily multilayered electrostatic problems has been presented in this article. Comparisons of the electric potentials between the FEM and DBEM analyses were discussed with respect to degenerate and non-degenerate designs in order to demonstrate the suitability and efficiency of computational method presented in this paper. It has been shown that the DBEM accompanied by subregion technology in the context of the present formulation is particularly suitable for the multilayered electrostatic problem with singularity arising from a degenerate boundary. For electrical engineering practices, since the major effort is model creation, the present DBEM, free from the development of an artificial boundary, has great potential for industrial applications.

\section{Appendix A}

In the derivation of dual equations, two alternatives can be applied to determine the Hadamard principal value as follows [12]:

(1) Trace operator first and differential operator second

$$
\begin{aligned}
& \text { HPV } \int_{B} M(s, x) u(s) \mathrm{d} B(s) \\
& =\frac{\partial}{\partial n_{x}}\left\{\mathrm{CPV} \int_{B} T(s, x) u(s) \mathrm{d} B(s)\right\} .
\end{aligned}
$$

For simplicity, constant element is adopted, i.e. $u(s)=1$, and (A1) reduces to

$$
\begin{aligned}
& \frac{\mathrm{d}}{\mathrm{d} x}\left\{\mathrm{CPV} \int_{a}^{c} \frac{-1}{(x-s)} \mathrm{d} s\right\} \\
& =\frac{\mathrm{d}}{\mathrm{d} x}\left\{\int_{a}^{x-\varepsilon} \frac{-1}{(x-s)} \mathrm{d} s+\int_{x+\varepsilon}^{c} \frac{-1}{(x-s)} \mathrm{d} s\right\} \\
& =\frac{1}{a-x}-\frac{1}{c-x}
\end{aligned}
$$

after using the Leibnitz rule.

(2) The differential operator first and trace operator second

$\operatorname{HPV} \int_{B} M(s, x) u(s) \mathrm{d} B(s)=\lim _{y \rightarrow x} \int_{B} M(s, y) u(s) \mathrm{d} B(s)$.

Similarly, constant element scheme can simplify (A2) into

$$
\begin{aligned}
& \lim _{y \rightarrow 0} \int_{a}^{c} \frac{1}{(x-s)^{2}+y^{2}} \mathrm{~d} s \\
& \quad=\lim _{y \rightarrow 0} \frac{1}{y}\left[\tan ^{-1}\left(\frac{y}{a-x}\right)-\tan ^{-1}\left(\frac{y}{c-x}\right)\right] \\
& \quad=\frac{1}{a-x}-\frac{1}{c-x}
\end{aligned}
$$

after using

$$
\lim _{\alpha \rightarrow 0} \frac{\tan ^{-1} \alpha}{\alpha}=1
$$

and

$$
\tan ^{-1} \alpha+\tan ^{-1} \frac{1}{\alpha}=\frac{\pi}{2}
$$

\section{Appendix B}

In dual BEM, the linear algebraic equations for an interior electrostatic problem discretized from the dual boundary integral equations can be written as $[12,13]$

$$
\begin{aligned}
& {\left[T_{p q}^{i}\right]\left\{\Phi_{q}\right\}=\left[U_{p q}^{i}\right]\{\partial \Phi / \partial n\}_{q}} \\
& {\left[M_{p q}^{i}\right]\left\{\Phi_{q}\right\}=\left[L_{p q}^{i}\right]\{\partial \Phi / \partial n\}_{q}}
\end{aligned}
$$


where $\left\{\Phi_{q}\right\}$ and $\{\partial \Phi / \partial n\}_{q}$ are the boundary potential and flux, and the subscripts $p$ and $q$ correspond to the labels of the collocation point and integration element, respectively. For the exterior electrostatic problem, we have $[12,13]$

$$
\begin{gathered}
{\left[T_{p q}^{t}\right]\left\{\Phi_{q}\right\}=\left[U_{p q}^{e}\right]\{\partial \Phi / \partial n\}_{q}} \\
{\left[M_{p q}^{e}\right]\left\{\Phi_{q}\right\}=\left[L_{p q}^{e}\right]\{\partial \Phi / \partial n\}_{q} .}
\end{gathered}
$$

The influence coefficients of the four square matrices $[U],[T]$, $[L]$ and $[M]$ can be represented as

$$
\begin{gathered}
U_{p q}=\operatorname{RPV} \int_{B_{q}} U\left(s_{q}, x_{p}\right) \mathrm{d} B\left(s_{q}\right) \\
T_{p q}=-\pi \delta_{p q}+\operatorname{CPV} \int_{B_{q}} T\left(s_{q}, x_{p}\right) \mathrm{d} B\left(s_{q}\right) \\
L_{p q}=\pi \delta_{p q}+\operatorname{CPV} \int_{B_{q}} L\left(s_{q}, x_{p}\right) \mathrm{d} B\left(s_{q}\right) \\
M_{p q}=\operatorname{HPV} \int_{B_{q}} M\left(s_{q}, x_{p}\right) \mathrm{d} B\left(s_{q}\right)
\end{gathered}
$$

where $B_{q}$ denotes the $q$ th element and $\delta_{p q}=1$ if $p=q$, otherwise it is zero. The explicit form will be derived in the following section. According to the dependence of the outnormal vectors in these four kernel functions for the interior and exterior electrostatic problems, their relationship can be easily found:

$$
\begin{gathered}
U_{p q}^{i}=U_{p q}^{e} \\
M_{p q}^{i}=M_{p q}^{e} \\
T_{p q}^{i}=-T_{p q}^{e} \quad \text { if } p \neq q ; \quad T_{p q}^{i}=T_{p q}^{e} \quad \text { if } p=q . \\
L_{p q}^{i}=-L_{p q}^{e} \quad \text { if } p \neq q ; \quad L_{p q}^{i}=L_{p q}^{e} \quad \text { if } p=q .
\end{gathered}
$$

\section{Appendix C}

(1) Subregion technology of BEM. Before studying the physical behaviour of interface between two media of electrostatic problems, the concept of subregion technology for singular BEM ([U] and $[T])$ or hypersingular BEM $([L]$ and $[M])$ was introduced first. From figure 2, a figure sketch of the multi-domain could be found, where $\left\{u_{c}^{1}\right\}$ is the boundary data of degenerate boundary $\left(C^{+}\right.$and $\left.C^{-}\right)$, and $\left\{u_{f}^{1}\right\},\left\{u_{f}^{2}\right\},\left\{t_{f}^{1}\right\}$, $\left\{t_{f}^{2}\right\}$ are the unknowns of the interface between subdomains 1 and 2. Since the degenerate boundary on $C^{+}$and $C^{-}$as shown in figure 2 results in double unknowns, Equation (8) or (9) can provide an additional equation by collocating the point $x$ on $C^{+}$and $C^{-}$. By dividing the domain into two subdomains (subdomains 1 and 2) and using singular BEM or hypersingular BEM influence matrices for each subdomain, we have the two equations from equation (8) or (9) as follows:

$$
\left[\begin{array}{cc}
T_{c c}^{1} & T_{c f}^{1} \\
T_{f c}^{1} & T_{f f}^{1}
\end{array}\right]\left\{\begin{array}{l}
u_{c}^{1} \\
u_{f}^{1}
\end{array}\right\}=\left[\begin{array}{ll}
U_{c c}^{1} & U_{c f}^{1} \\
U_{f c}^{1} & U_{f f}^{1}
\end{array}\right]\left\{\begin{array}{l}
t_{c}^{1} \\
t_{f}^{1}
\end{array}\right\}
$$

and

$$
\left[\begin{array}{cc}
T_{c c}^{2} & T_{c f}^{2} \\
T_{f c}^{2} & T_{f f}^{2}
\end{array}\right]\left\{\begin{array}{l}
u_{c}^{2} \\
u_{f}^{2}
\end{array}\right\}=\left[\begin{array}{cc}
U_{c c}^{2} & U_{c f}^{2} \\
U_{f c}^{2} & U_{f f}^{2}
\end{array}\right]\left\{\begin{array}{c}
t_{c}^{2} \\
t_{f}^{2}
\end{array}\right\}
$$

where the superscripts 1 and 2 are the labels of the subdomains and the subscripts $c$ and $f$ denote the complementary and interface sets for $u$ and $t$, respectively. Since the unknown pairs of $\left\{u_{f}^{1}\right\},\left\{u_{f}^{2}\right\},\left\{t_{f}^{1}\right\}$ and $\left\{t_{f}^{2}\right\}$ are introduced in the artificial boundary as shown in figure 2 , two constraints of the continuity and equilibrium conditions $\left(\left\{u_{f}^{1}\right\}=\left\{u_{f}^{2}\right\}\right.$ and $\left.\left\{t_{f}^{1}\right\}=-\left\{t_{f}^{2}\right\}\right)$ are necessary for the interface if $\varepsilon_{1}=\varepsilon_{2}$. By assembling (C1) and (C2) with two constraints of the continuity and equilibrium conditions for the interface, we have

$\left[\begin{array}{ccc}U_{c c}^{1} & U_{c f}^{1} & 0 \\ U_{f c}^{1} & U_{f f}^{1} & 0 \\ 0 & -U_{c f}^{2} & U_{c c}^{2} \\ 0 & -U_{f f}^{2} & U_{f c}^{2}\end{array}\right]\left\{\begin{array}{l}t_{c}^{1} \\ t_{f}^{1} \\ t_{c}^{2}\end{array}\right\}=\left[\begin{array}{ccc}T_{c c}^{1} & T_{c f}^{1} & 0 \\ T_{f c}^{1} & T_{f f}^{1} & 0 \\ 0 & T_{c f}^{2} & T_{c c}^{2} \\ 0 & T_{f f}^{2} & T_{f c}^{2}\end{array}\right]\left\{\begin{array}{l}u_{c}^{1} \\ u_{f}^{1} \\ u_{c}^{2}\end{array}\right\}$.

After solving (C3), all the unknowns $\left\{t_{c}^{1}\right\},\left\{t_{c}^{2}\right\},\left\{t_{f}^{1}\right\}$ and $\left\{u_{f}^{1}\right\}$ can be obtained, and $\left\{u_{f}^{2}\right\}$ and $\left\{t_{f}^{2}\right\}$ also can be calculated from the two constraints of the continuity and equilibrium conditions for the interface.

(2) Boundary conditions for electrostatic fields of the interface between two media. If there is an interface between two general media shown in figure 3, the normal component of $\boldsymbol{D}$ field is discontinuous across an interface where a surface charge exists - the amount of discontinuity being equal to the surface charge density. When two dielectrics are in contact with no free charges at the interface, $\rho_{s}=0$, we have

$$
\varepsilon_{1} \boldsymbol{E}_{1 n}=\varepsilon_{2} \boldsymbol{E}_{2 n}
$$

In addition, the electric potential and the tangential component of an $\boldsymbol{E}$ field are all continuous across an interface [1,2].

(3) Subregion technology for multilayered electrostatic problems. For multilayered domain $\left(\varepsilon_{1} \neq \varepsilon_{2}\right)$ shown in figure 3, by assembling (C1) and (C2) with two constraints of the continuity and boundary conditions $(\mathrm{C} 4)$ for the interface $\left(\left\{u_{f}^{1}\right\}=\left\{u_{f}^{2}\right\}\right.$ and $\left\{t_{f}^{1}\right\}=-R\left\{t_{f}^{2}\right\}$, where $R=\varepsilon_{1} / \varepsilon_{2}$ is the ratio of permittivity between subdomains 1 and 2), we have

$$
\left[\begin{array}{ccc}
U_{c c}^{1} & U_{c f}^{1} & 0 \\
U_{f c}^{1} & U_{f f}^{1} & 0 \\
0 & -R U_{c f}^{2} & U_{c c}^{2} \\
0 & -R U_{f f}^{2} & U_{f c}^{2}
\end{array}\right]\left\{\begin{array}{c}
t_{c}^{1} \\
t_{f}^{1} \\
t_{c}^{2}
\end{array}\right\}=\left[\begin{array}{ccc}
T_{c c}^{1} & T_{c f}^{1} & 0 \\
T_{f c}^{1} & T_{f f}^{1} & 0 \\
0 & T_{c f}^{2} & T_{c c}^{2} \\
0 & T_{f f}^{2} & T_{f c}^{2}
\end{array}\right]\left\{\begin{array}{l}
u_{c}^{1} \\
u_{f}^{1} \\
u_{c}^{2}
\end{array}\right\} .
$$

After solving (C5), all the unknowns $\left\{t_{c}^{1}\right\},\left\{t_{c}^{2}\right\},\left\{t_{f}^{1}\right\}$ and $\left\{u_{f}^{1}\right\}$ can be obtained, and $\left\{u_{f}^{2}\right\}$ and $\left\{t_{f}^{2}\right\}$ also can be calculated from the two constraints of the continuity and boundary conditions for the interface. 


\section{References}

[1] Cross J A 1987 Electrostatics: Principles, Problems and Applications (Bristol: Adam Hilger)

[2] Haus H A and Melcher J R 1989 Elctromagnetic Fields and Energy (Englewood Cliffs, NJ: Prentice Hall)

[3] Liu D C 2003 On the theory of the modal expansion method for the EM fields in side a closed volume J. Phys. D: Appl. Phys. 36 1629-33

[4] Sadiku M N O 1992 Numerical Techniques in Electromagnetics (Boca Raton, FL: CRC Press)

[5] Jin J 2002 The Finite Element Method in Electromagnetics (New York: Wiely)

[6] Aliabadi M H 2002 The Boundary Element Method (New York: Wiely)

[7] Senturia S D, Aluru N and White J 1997 Simulating the behavior of MEMS devices: computational methods and needs IEEE Comput. Sci. Eng. Mag. 30-43

[8] Wiersig J 2003 Boundary element method for resonances in dielectric microcavities J. Opt. A: Pure Appl. Opt. 5 53-60

[9] Ye W, Mukherjee S and MacDonald N C 1998 Optimal shape design of an electrostatic comb drive in microelectromechanical systems IEEE J. Microelectromech. Syst. 7 16-26

[10] Chakravorti S and Steinbigler H 2000 Boundary element studies on insulator shape and electric field around HV insulators with or without pollution IEEE Trans. Dielectr. Electr. Insul. 7 167-76

[11] Chyuan S W, Liao Y S and Chen J T 2004 Computational study of variations in gap size for the electrostatic levitating force of MEMS device using dual BEM Microelectron. J. at press
[12] Liao Y S, Chyuan S W and Chen J T 2004 An alternatively efficient method (DBEM) for simulating the electrostatic field and levitating force of a MEMS combdrive J. Micromech. Microeng. 14 1258-69

[13] Chyuan S W, Liao Y S and Chen J T 2003 An efficient method for solving electrostatic problems IEEE Comput. Sci. Eng. Mag. 52-8

[14] Rao S M, Sarkar T K and Harrington R F 1984 The electrostatic field of conducting bodies in multiple dielectric media IEEE Trans. Microwave Theory Tech. 32 1441-8

[15] Wei C, Harrington R F, Mautz J R and Sarkar T K 1984 Multiconductor transmission lines in multilayered dielectric media IEEE Trans. Microwave Theory Tech. 32 439-50

[16] Li K, Atsuki K and Hasegawa T 1997 General analytical solutions of static Green's functions for shielded and open arbitrarily multilayered media IEEE Trans. Microwave Theory Tech. 45 2-8

[17] Cangellaris A and Yang L 2001 Rapid calculation of electrostatic Green's functions in layered dielectrics IEEE Trans. Magn. 37 3133-6

[18] Smith P M 2001 Dyadic Green's functions for multi-layer SAW substrates IEEE Trans. Ultrason., Ferroelectr. Freq. Control 48 171-9

[19] Tan E L 2002 Electrostatic Green's functions for planar multilayered anisotropic media IEE Proc., Microw. Antennas Propag. 149 78-83

[20] Chen J T and Lin S R 2002 On the rank-deficiency problems in boundary integral formulation using the Fredholm alternative theory and singular value decomposition technique Proc. 5th World Congress on Computational Mechanics (Vienna, 7-12 July)

[21] I-DEAS User's Guide, Finite Element Modeling, SDRC, 1990 